11.1

\title{
Фурье-спектры амплитудных вариаций суперпозиции сигналов навигационных спутников вблизи поверхностей с различными свойствами
}

\author{
(C) E.B. Кузьмин ${ }^{1}$, А.В. Сорокин ${ }^{2}$ \\ ${ }^{1}$ Сибирский федеральный университет, Красноярск, Россия \\ 2 Федеральный исследовательский центр „Красноярский научный центр СО РАН“, Красноярск, Россия \\ E-mail: ekuzmin@sfu-kras.ru, sorav@iph.krasn.ru
}

Поступило в Редакцию 9 апреля 2021 г.

В окончательной редакции 22 мая 2021 г.

Принято к публикации 8 июня 2021г.

Представлены экспериментально зарегистрированные амплитудно-временны́е зависимости интерференционной суперпозиции сигналов навигационных спутников и их копий, отраженных границами разделов сред атмосфера-вода и атмосфера-суша. Получены фурье-спектры данных зависимостей. Установлено, что при различных электрофизических свойствах отражающих поверхностей наблюдается появление заметных особенностей спектров.

Ключевые слова: ГНСС-рефлектометрия, поверхность, интерференция, амплитудно-временна́я зависимость, фурье-спектр.

DOI: 10.21883/PJTF.2021.18.51468.18818

Глобальные навигационные спутниковые системы (ГНСС) активно используются для решения различных практических и научных задач [1]. В частности, непрерывно излучаемые сигналы навигационных спутников (HC) применяются для получения данных о характеристиках земных покровов, что позволяет осуществлять бесконтактный мониторинг параметров различных поверхностей [2-6]. Сигналы НС взаимодействуют с поверхностями земных покровов, в результате чего формируется интерференционное поле, образуемое суперпозицией прямого сигнала и его копий, отраженных границами разделов сред. Движение НС приводит к перемещению площадки отражения. Таким естественным образом производится „сканирование“ поверхностей в окрестности антенны. Приемник сигналов ГНСС производит типовые измерения и регистрирует числовые значения амплитудно-временны́х зависимостей (AB3) интерференционной суперпозиции процессов в точке приема. Последующий спектральный анализ AB3, примененный авторами, расширяет возможности ГНСС-рефлектометрии исследуемых поверхностей земных покровов.

В настоящей работе проведена регистрация АВ3 при распространении сигналов НС в атмосфере, вблизи водных поверхностей и почвенных покровов. Условия регистрации были аналогичными представленным в работе [6].

На рис. 1-3 приведены экспериментально зарегистрированные отсчеты $x=x(n)$ AB3 $(a)$ и рассчитанные на их основе модули фурье-спектров, нормированные к их максимальным значениям: $X=|X(f)| /\left|X_{\max }(f)\right|(b)$. Кривые 1 соответствуют АВ3, полученным в результате приема сигналов НС ГЛОНАСС. Кривые 2 получены при приеме сигналов HC GPS. Символами $n$ обозначены временны́е отсчеты, следующие с шагом $1 \mathrm{~s}$. Спектры $X(f)$ получены на основе дискретного преобразования Фурье [7] отсчетов $x(n)$.

Зависимости, показанные на рис. 1, $a$, зарегистрированы при использовании антенны с правой круговой поляризацией, ориентированной в верхнюю полусферу. Такие условия наблюдения авторы условно называют случаем распространения в „свободном пространстве“, поскольку вклад отраженных сигналов, образующих интерференционную суперпозицию, отсутствует либо мал. Данный случай необходим как „индикатор“ отсутствия отражающей поверхности.

Кривые, показанные на рис. 2, $a$ и 3, $a$, зарегистрированы при использовании дипольной антенны, расположенной перпендикулярно относительно горизонтальной плоскости. Такой способ ориентирован на учет вклада в интерференционную суперпозицию процессов, отраженных или рассеянных различными поверхностями. Рис. 2 соответствует случаю прохождения площадки отражения по почвенному покрову и сегменту водной поверхности. Рис. 3 отвечает случаю регистрации АВЗ в окрестности исключительно водной поверхности.

Как видно из рис. 1, для случая „свободного пространства“ зарегистрированные АВ3 и полученные фурьеспектры не имеют особенностей, которые возникают при одновременном присутствии прямых и отраженных горизонтальной поверхностью сигналов НС. Это объясняется тем, что атмосферные неоднородности в L1-диапазоне вносят достаточно малый вклад в регистрируемую АВЗ суммарного интерференционного процесcа. 

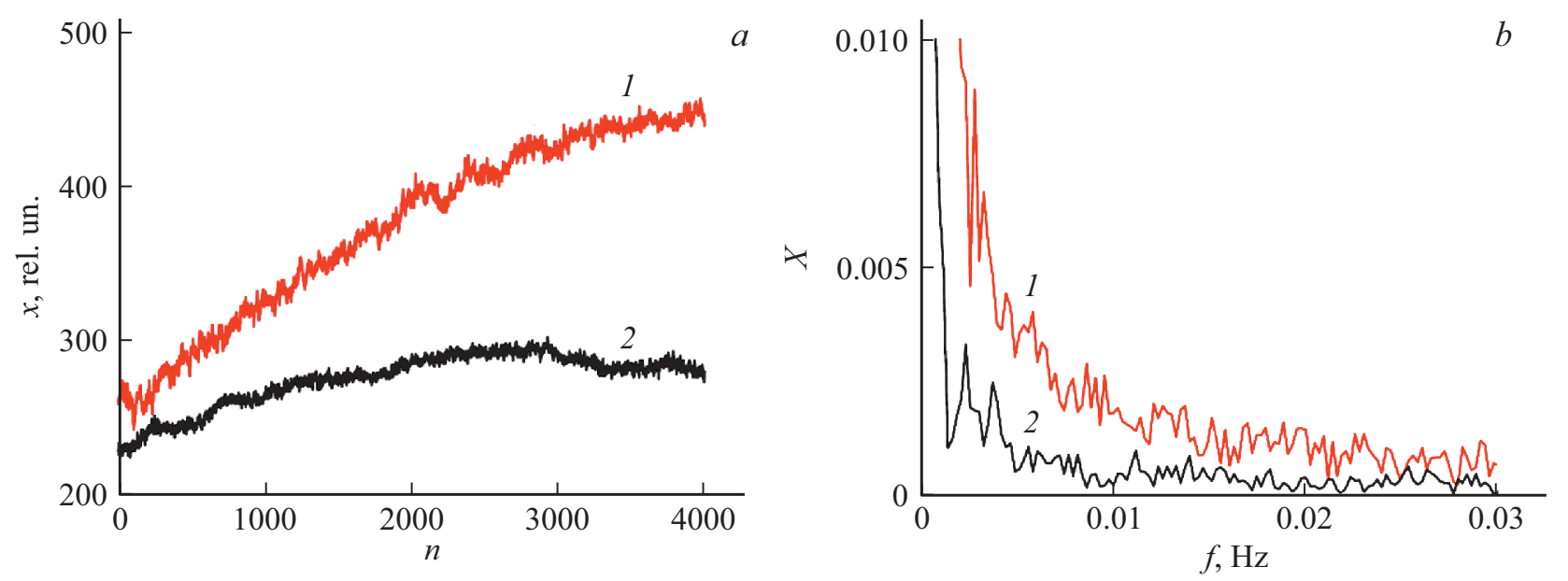

Рис. 1. Амплитудно-временнб́е зависимости $(a)$ и соответствующие им нормированные фурье-спектры $(b)$. Случай распространения в „свободном пространстве“.
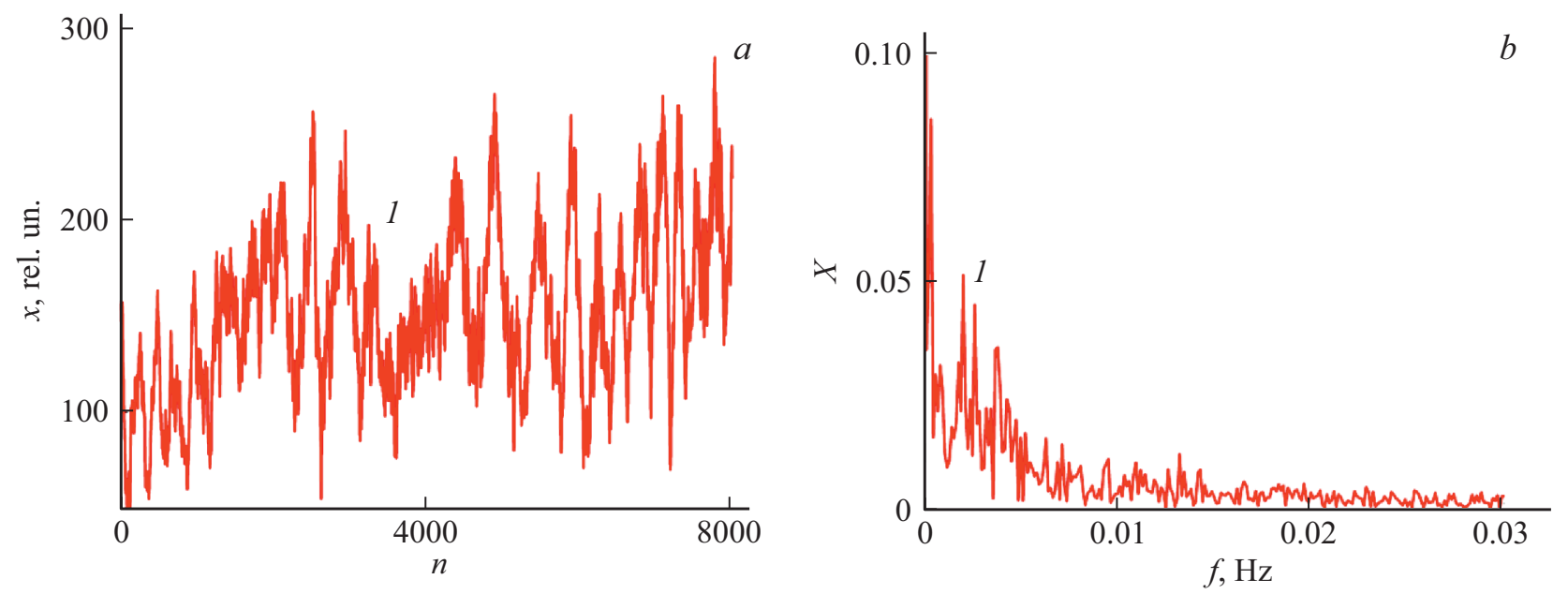

Рис. 2. Амплитудно-временна́я зависимость $(a)$ и соответствующий ей нормированный фурье-спектр $(b)$. Преимущественно почвенный покров и сегмент водной поверхности.

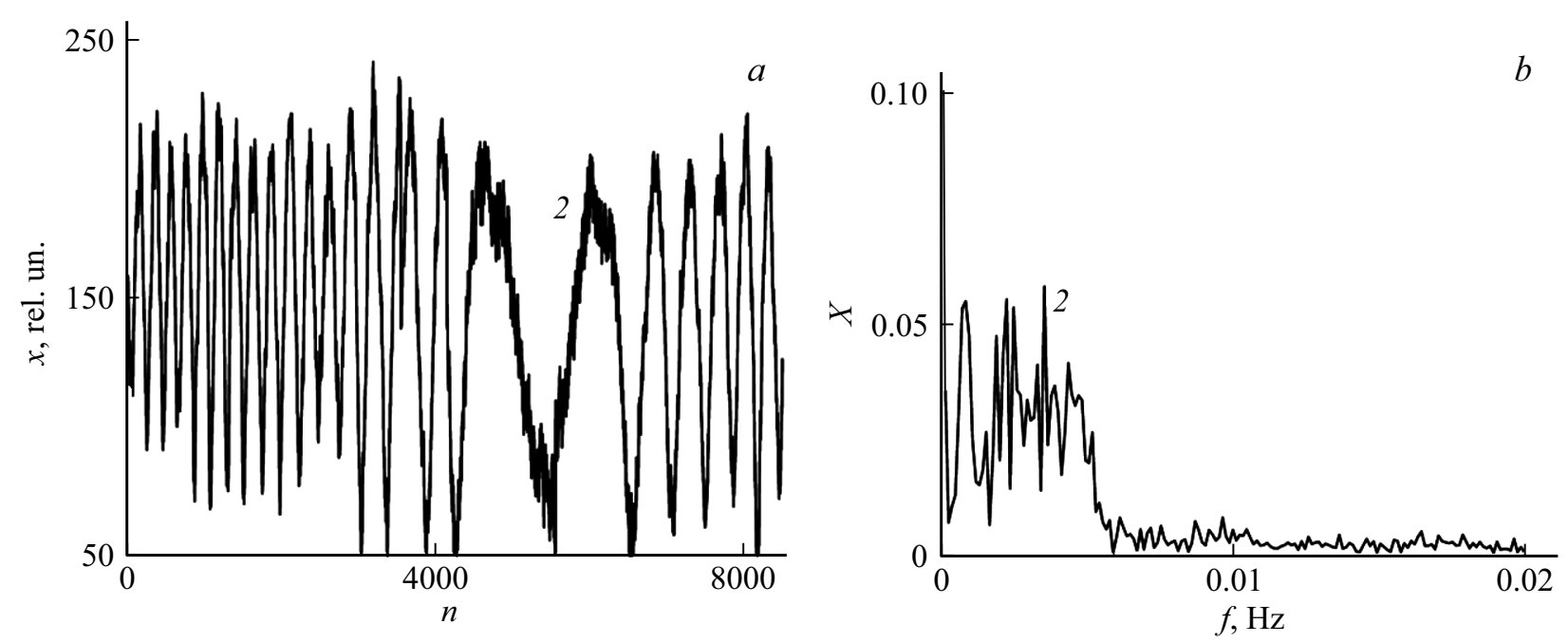

Рис. 3. Амплитудно-временна́я зависимость $(a)$ и соответствующий ей нормированный фурье-спектр $(b)$. Водная поверхность. 
Из рис. 2, 3 видно, что АВ3 и фурье-спектры обладают выраженными особенностями, которые различаются в зависимости от типа поверхности (почва, вода). Это объясняется наличием в интерференционной суперпозиции компоненты сигнала, отраженного границами раздела сред атмосфера-вода и атмосфера-суша. Из рис. 1 видно, что поведение кривых оказывается подобным при регистрации АВ3 по сигналам обеих систем. Кроме того, из представленных рисунков видно (фрагменты $a$ ), что значения всех зарегистрированных АВ3 находятся в границах интервала $0<x(n)<500$. При этом величины нормированных спектров для случая „свободного пространства“ (рис. 1,b) приблизительно на порядок меньше, чем в случаях наличия отраженного сигнала (рис. 2, $b$ и $3, b$ ).

Таким образом, в работе получены экспериментальные амплитудно-временны́е зависимости интерференционной суперпозиции сигналов НС, принимаемых в условиях наличия и отсутствия отражающих поверхностей. Полученные фурье-спектры зарегистрированных зависимостей имеют визуально различимые особенности, изменяющиеся для различных условий приема сигналов. Показано, что при рассмотрении АВЗ для случая распространения сигналов НС в атмосфере фурье-спектры имеют монотонно убывающее поведение с некоторыми „выбросами“ на начальном участке. Установлено, что в случае приема сигналов НС в окрестности отражающих поверхностей в спектрах АВ3 наблюдаются явные изменения. Спектры приобретают многочисленные „всплески“ и „провалы“, становятся „расширенными“ и занимают бо́льшую полосу частот в сравнении с вариантом распространения сигналов НС только в атмосфере. Указанное расширение особенно заметно для случая приема сигналов НС вблизи водной поверхности: зарегистрированные АВ3 и их спектры подобны соответственно временны́м и спектральным диаграммам сигналов с внутриимпульсной частотной модуляцией. Представленные авторами примеры наглядно демонстрируют, что фурье-спектры АВЗ интерференционной суперпозиции сигналов НС представляются востребованным инструментом для повышения эффективности и расширения возможностей ГНСС-рефлектометрии при изучении свойств различных поверхностей.

\section{Конфликт интересов}

Авторы заявляют, что у них нет конфликта интересов.

\section{Список литературы}

[1] Springer handbook of global navigation satellite systems, ed. by P.J.G. Teunissen, O. Montenbruck (Springer International Publ., 2017).

[2] В.Б. Кашкин, В.И. Кокорин, В.Л. Миронов, С.В. Сизасов, Радиотехника и электроника, 51 (7), 825 (2006).

[3] S. Jin, E. Cardellach, F. Xie, GNSS remote sensing (Springer, Dordrecht-Heidelberg-N.Y.-London, 2014).
[4] М.И. Михайлов, К.В. Музалевский, В.Л. Миронов, Современные проблемы дистанционного зондирования Земли из космоса, 14 (2), 167 (2017). DOI: 10.21046/2070-7401-2017-14-2-167-174

[5] А.М. Падохин, Г.А. Курбатов, М.О. Назаренко, В.Е. Смолов, Вестн. МГУ. Сер. 3. Физика. Астрономия, № 4, 80 (2018).

[6] Д.С. Макаров, А.В. Сорокин, Д.В. Харламов, Сиб. журн. науки и технологий, 20 (1), 8 (2019). DOI: $10.31772 / 2587-6066-2019-20-1-8-19$

[7] С.Л. Марпл, мл. Цифровой спектральный анализ и его приложсения, пер. с англ. (Мир, М., 1990). 